Relations industrielles

Industrial Relations

\title{
Le Bill 36 et le syndicalisme de cadre
}

\section{Claude D'Aoust}

Volume 25, numéro 3, 1970

URI : https://id.erudit.org/iderudit/028160ar

DOI : https://doi.org/10.7202/028160ar

Aller au sommaire du numéro

Éditeur(s)

Département des relations industrielles de l'Université Laval

ISSN

0034-379X (imprimé)

1703-8138 (numérique)

Découvrir la revue

Citer cet article

D’Aoust, C. (1970). Le Bill 36 et le syndicalisme de cadre. Relations industrielles /

Industrial Relations, 25(3), 617-620. https://doi.org/10.7202/028160ar

Tous droits réservés (C) Département des relations industrielles de l'Université Laval, 1970
Ce document est protégé par la loi sur le droit d'auteur. L’utilisation des services d'Érudit (y compris la reproduction) est assujettie à sa politique d'utilisation que vous pouvez consulter en ligne.

https://apropos.erudit.org/fr/usagers/politique-dutilisation/ 


\section{LE BILL 36 ET LE SYNDICALISME DE CADRE}

\section{Claude D'Aoust}

Le législateur vient d'amender à nouveau le Code du Travail ${ }^{1}$. Un alinéa est ainsi ajouté à l'article 20 du Code, réglant le cas des associations de cadres en activité à l'Hydro-Québec et à la Ville de Montréal. Ces associations sont déclarées accréditées par la loi, comme si l'accréditation avait été accordée par un commissaire-enquêteur.

Ce n'est pas par son originalité que cet amendement nous intéresse : le procédé avait déjà été utilisé auparavant ${ }^{2}$. Il tire plutôt son intérêt du fait qu'il éclaircit certaines questions que l'on pouvait se poser quant au statut juridique des syndicats groupant en totalité ou en partie des cadres. L'amendement est également digne d'attention en ce qu'il indique l'évolution et l'orientation de la pensée du législateur à l'égard du syndicalisme de cadres.

\section{StatuT JURIdique DES SYNDICATS DE CADRES ET DES SYNDicats MIXTES.}

On sait que le Bill 50 a fait disparaitre du code l'association reconnue ${ }^{3}$, et d'après les notes explicatives du Bill 36 , l'amendement corrigerait partiellement cette disparition. C'est ce qu'indique également la date du 2 août 1969 qui apparaît à l'article $1^{4}$. Mais je ne suis pas certain que cette relation soit juste.

En ce qui concerne les associations formées exclusivement de cadres, le statut qui leur était accordé par l'employeur ne constituait pas à mon avis une reconnaissance au sens du Code. L'article 1, paragraphe c) définissait l'association reconnue comme un groupement de «salariés ». Et la définition de salarié, à l'article 1, paragraphe m), excluait justement

* Professeur au Département de relations industrielles de l'Université de Montréal.

1 Loi modifiant le Code du Travail, Bill 36, sanctionné le 17 juillet 1970. Le texte de la loi est reproduit en appendice.

2 Loi de la Fonction Publique, 13-14 Eliz. II, S. Qué., 1965, chap. 14, art. 69 à 71 .

Pour des cas analogues, voir : Loi assurant le droit de l'enfant à l'éducation et instituant un nouveau régime de convention collective dans le secteur scolaire, 15-16 Eliz. II, S. Qué., 1966-67, chap. 63, art. 13 à 15 et 31 ; Loi concernant le régime syndical applicable à la Sûreté du Québec, 16-17 Eliz. II, S. Qué., chap. 19, art. 2 à 4 ; Loi des relations du travail dans l'industrie de la construction, 16-17 Eliz. II, S. Qué., 1967-68, chap. 45, art. 3 à 8.

3 Loi modifiant le Code du Travail, Lois du Québec, 1969, chap. 47.

4 L'article 2, parag. b) du Bill 50, abolissant l'association reconnue, est entré en vigueur à la proclamation partielle du 2 août 1969. 
les cadres ${ }^{5}$. En conséquence, un tel syndicat ne pouvait être reconnu au sens du Code pas plus qu'il ne pouvait être accrédité. Je n'ignore pas que cette opinion n'est pas universellement partagée. Mais si un syndicat de cadres pouvait être accrédité selon le Code, pourquoi le législateur a-t-il jugé nécessaire de voter le Bill 36 ? Ayant perdu leur statut d'association reconnue par suite de l'adoption du Bill 50, ces associations auraient pu présenter une requête en accréditation. A mon avis, le Bill 36 , c'est l'exception qui confirme la règle de l'exclusion générale des cadres de l'ensemble du Code du Travail ${ }^{6}$. En outre, l'exception ainsi créée confirme la jurisprudence ancienne de la C.R.T. sur ce sujet 7 .

Quant aux syndicats groupant à la fois des salariés au sens du Code et des cadres - que j'appelle syndicats mixtes - leur statut juridique était encore plus imprécis. Ils ne pouvaient réquérir l'accréditation sans se voir amputer une partie de leurs effectifs par la C.R.T. Si l'employeur acceptait librement de négocier avec un tel syndicat, cette « reconnaissance » en faisait-elle une association reconnue au sens de l'article 1, paragraphe c) ? Si oui, la reconnaissance valait-elle pour toutes les catégories de membres ou seulement pour les membres salariés au sens de l'article $1, \mathrm{~m}$ ) L'entente négociée était-elle une convention collective au sens du Code ? Si oui, l'article 55 s'appliquait-il à toutes les catégories de membres?

Le Bill 36 jette peu de lumière sur ces questions, sauf d'indiquer que l'accréditation par la voie normale ne permettrait pas à un syndicat mixte de conserver son intégrité.

L'ORIENTATION DE LA PENSÉE DU LÉGISLATEUR.

Par le Bill 36, le législateur reconnaît positivement le syndicalisme de cadres, de façon limitée il est vrai, de la même manière que la Loi des Relations Ouvrières reconnaissait positivement le syndicalisme industriel ${ }^{8}$. On peut donc prévoir que de nouvelles étapes seront franchies, pour en arriver à une acceptation générale de ce genre de syndicalisme. En tout cas, les déclarations d'intention du gouvernement vont en ce sens ${ }^{9}$.

D'autre part, le Bill 36 confère l'accréditation à des syndicats mixtes. $\mathrm{Si}$ de tels syndicats ont pu exister et fonctionner à $1^{1} H y d r o-Q u e ́ b e c$ et à

5 Le mot «cadres» est employé ici dans un sens très large et inclut less gérants, surintendants et contremaîtres.

$6 \mathrm{La}$ question de savoir si le Bill 36 s'applique réellement à un syndicat formé exclusivement de cadres est discutée en appendice.

$7 \mathrm{La}$ portée d'un amendement sur la jurisprudence a été commentée brièvement par le juge Pigeon dans l'arrêt Steinberg's Ltée v. Le 'Comité Paritaire de l'alimentation au détail, Région de Montréal et al., (1968) R.C.S. 971, pp. 979.-80.

8 S.R.Q., 1941, chap. 162 A.

9 Voir les commentaires du ministre Laporte lors de l'étude du projet de loi par l'Assemblée Nationale, dans Journal des Débats, Première session, 29e Législature, Vol. 10, No. 19, 16 juillet 1970, pp. 1203-5. 
la Ville de Montréal, il n'est pas prouvé que cette formule soit applicable de façon générale à toutes les entreprises. Cela est d'autant plus vrai que les syndicats en question ont un autre caractère particulier, en plus de leur composition mixte, les distinguant des syndicats ordinaires. $\mathrm{Ce}$ sont en effet des syndicats d'ingénieurs; or on sait que les travailleurs professionnels diffèrent grandement des autres catégories de travailleurs. Au surplus, l'existence de ces syndicats remonte à cinq ou six ans seulement. Si l'on ajoute à cela que l'Hydro-Québec et la Ville de Montréal étant des services publics ne peuvent être considérés comme des employeurs typiques, l'expérience des syndicats mixtes au Québec est une base fragile pour la rédaction d'une nouvelle législation.

\section{APPENDICE I}

BILL 36

Loi modifiant le Code du travail

«SA MAJESTÉ, de l'avis et du consentement de l'Assemblée nationale du Québec, décrète ce qui suit:

1. L'article 20 du Code du travail (Statuts refondus, 1964, chapitre 141), remplacé par l'article 9 du chapitre 47. des lois de 1969 et modifié par l'article 9 du chapitre 48 des lois de 1969, est de nouveau modifié en ajoutant, à la fin, l'alinéa suivant :

«Les associations qui étaient reconnues par la Commission hydroélectrique de Québec ou la Ville de Montréal le 2 août 1969 pour représenter des groupes de personnes comprenant en totalité ou en partie des gérants, surintendants, contremaîtres ou représentants de leur employeur dans ses relations avec ses salariés et qui, à cette date ou dans l'année précédant cette date, étaient à leur égard parties signataires à une entente collective de travail, sont à compter du 17 juillet 1970 des associations accréditées à leur égard comme si l'accréditation avait été accordée par un commissaire-enquêteur; à compter du 1er janvier 1972, ces associations sont soumises aux règles générales alors applicables à l'accréditation. 》

2. La présente loi entre en vigueur le jour de sa sanction.

\section{APPENDICE II}

Selon le Ministre du Travail, le Bill 36 est une loi d'exception qui vise deux associations seulement : celles des ingénieurs de l'Hydro-Québec et de la Ville de Montréal ${ }^{10}$. Cela à mon avis n'est pas certain et voici pourquoi :

a) les deux syndicats d'ingénieurs ne sont pas désignés par leur nom ;

10 Journal des Débats, Première session, 29e Législature, Vol. 10, No. 17, 14 juillet 1970, pp. 950-51, et Vol. 10, No 19, 16 juillet 1970, p. 1201 et seq. 
b) les termes utilisés sont assez larges pour inclure d'autres syndicats de l'Hydro et de la Ville reconnus au 2 août 1969 s'il en existe;

c) le Bill parle d'associations reconnues \& pour représenter des groupes de personnes comprenant EN TOTALITE OU EN PARTIE des gérants, surintendants, contremaîtres ou représentants de leur employeur dans ses relations avec ses salariés. . . . ${ }^{11}$. Or, les deux syndicats d'ingénieurs visés sont tous deux mixtes i.e. formés EN PARTIE de cadres ;

d) En plus d'une reconnaissance antérieure au 2 août 1969, une association doit également avoir été partie à une convention à cette date, ou dans les douze mois précédents, pour jouir de l'accréditation accordée par le Bill 36.

Selon les renseignements que j'ai pu obtenir, il existe à la Ville de Montréal une association de contremaîtres qui négocie depuis 1946, qui est formée EN TOTALITE de cadres, et qui était partie à une convention le 2 août 1969. Il semble donc que cette association soit maintenant accréditée par le Bill 36.

\section{ÉPILOGUE SUR LES DIFFICULTÉS DE LA RÉDACTION LÉGISLATIVE}

Il est bien connu que la rédaction des lois est un art difficile. Le Bill 36 nous en donne une autre illustration.

Ce Bill a comme objet d'amender le Code du Travail. Il importait donc de faire particulièrement attention à l'emploi de mots utilisés (ou ayant déjà été utilisés) dans le Code ${ }^{12}$. Or le Bill vise des associations de cadres ou des syndicats mixtes RECONNUS avant le 2 juin 1969. S'il est juste de penser qu'un syndicat de cadres ne pouvait constituer une «association reconnue » au sens de l'ancien article 1, paragraphe c), il s'ensuivrait qu'aucune association existante ne serait de fait affectée par la nouvelle loi. Je crois cependant que les lignes qui suivent (soit les lignes 4 à 7) clarifient l'intention du législateur. Cette faute ne serait donc qu'apparente.

D'autre part, la terminologie utilisée plus loin confirme l'opinion qu'un syndicat de cadres ou un syndicat mixte ne pouvait, même avant la disparition des associations reconnues, signer une convention collective au sens du Code. En effet, le Bill 36 utilise les termes «entente collective de travail $\gg$, et « collective labour arrangement » dans la version anglaise. Il faut donc présumer que le législateur voulait * introduire une distinction ou une nuance $\gg 13$.

11 J'ai ajouté les majuscules.

12 Sur cette question, voir : Louis-Philippe PIGEON, Rédaction et interprétation des lois, Imprimeur de la Reine, Québec, 1965, pp. 28-29 et p. 35.

13 Ibid., p. 28. 